\title{
A Numerical Renormalization Group approach to Green's Functions for Quantum Impurity Models
}

\author{
Robert Peters and Thomas Pruschke \\ Institut für Theoretische Physik, Universität Göttingen, D-37077 Göttingen, Germany \\ Frithjof B. Anders \\ Institut für Theoretische Physik, Universität Bremen, P.O. Box 330 440, D-28334 Bremen, Germany
}

(Dated: 07-19-2006)

\begin{abstract}
We present a novel technique for the calculation of dynamical correlation functions of quantum impurity systems in equilibrium with Wilson's numerical renormalization group. Our formulation is based on a complete basis set of the Wilson chain. In contrast to all previous methods, it does not suffer from overcounting of excitation. By construction, it always fulfills sum rules for spectral functions. Furthermore, it accurately reproduces local thermodynamic expectation values, such as occupancy and magnetization, obtained directly from the numerical renormalization group calculations.
\end{abstract}

PACS numbers:

\section{INTRODUCTION}

The understanding of quantum impurity systems became one the cornerstones of condensed matter theory during the last decade. Quantum impurity systems appear at the heart of a variety of different physical problems. Traditionally, they were used to describe the interaction of magnetic impurities with a metallic host $\frac{1}{1}$ Nowadays, quantum impurity systems play a fundamental role in our understanding of the low temperature properties of single-electron transistors ${ }^{2.3}$ and the tunneling spectroscopy of adatoms on metal surfaces $\frac{4.5}{4}$ The basic structure common to all such systems is a mesoscopic subsystem or device - such as a quantum dot, an organic donor-acceptor molecule or an adatom - coupled to a continuum of states that can be represented by noninteracting particles of either fermionic or bosonic nature. Typical realizations are models like the single impurity Anderson model,,$\frac{6}{n}$ where the continuum of states is described by free fermions, or the spin-boson model, ${ }^{7}$ where the discrete orbitals interact with a bosonic bath. In addition, within the dynamical mean-field theory ${ }^{8.9}$ or its cluster extensions $\frac{10}{b}$ lattice models for strongly correlated fermions have been mapped onto quantum impurity problems embedded in a fictitious, self-consistent bath. Obtaining a self-consistent solution for these theories requires very accurate determination of local Green's functions.

Wilson's numerical renormalization group (NRG) approach $^{11}$ to quantum impurity problems is one of the most powerful and flexible ways for accurately calculating thermodynamic properties of quantum impurity systems. In addition, it provides a deep insight into the underlying physics through the analysis of the fixed point Hamiltonians $, 12,13.14$ The key ingredient is a logarithmic discretization of the bath continuum, resulting in a well defined hierarchy of energy or temperature scales. The discretized model is then iteratively diagonalized and the basis set truncated, retaining only those states with low lying energies after each step. Each iteration represents a certain temperature $T$, and all thermodynamic properties are determined for that particular $T, 11,12$ For calculating dynamical properties 15.16 .17 .18 .19 three fundamental problems arise: (i) how to recover the continuum limit from a discretized spectrum, (ii) how to obtain dynamical information on all energies scale at arbitrarily low temperature in such away that (iii) spectral sum rules are always fulfilled and thermodynamics expectation values are reproduced exactly independent of how many states are kept after each NRG step.

In this paper, we will derive an exact analytical expression for arbitrary dynamical correlation functions which solves this problems. It is based upon the recent identification of a complete basis set of the Wilson chain, which is also an approximate eigenbasis of the NRG Hamiltonian ${ }^{20,21}$ We will show that this complete basis set automatically ensures that spectral sum rules are fulfilled exactly and thermodynamic expectation values reproduced accurately by the spectral functions. We furthermore explicitly demonstrate that our novel approach yields spectral functions which are largely insensitive to the number of eigenstates kept in each NRG iteration, thus improving the applicability of the NRG to multi-orbital and multi-site impurity problems and consequently also to multi-band and cluster DMFT problems, because the number of NRG states needed can be significantly reduced without loosing accuracy. In addition, phenomenological patching algorithms typically employed to combine excitations from different NRG iterations ${ }^{15}$ become obsolete, since we rigorously identify which excitations actually contribute at which Wilson shell.

The paper is organized as follows. In the next section we will briefly review the basic theoretical concepts and derive the complete basis set. We furthermore show how this complete basis set can be used to calculate dynamical correlation functions. Section III is devoted to a detailed discussion of results for the simplest and most important 
quantum impurity model, the single impurity Anderson model. We will explicitly compare our new method to the standard implementations. A summary and outlook in section IV will conclude the paper. Proofs for our claim that sum rules and spectral averages are automatically respected within this formulation are given in the appendix.

\section{THEORY}

The NRG is a very powerful tool for accurately calculating equilibrium properties of quantum impurity models. Originally developed for treating the singlechannel, single-impurity Kondo Hamiltonian, 11.22 this non-perturbative approach was successfully extended to the Anderson impurity model,,$\frac{6.12 .13}{1}$ the two-channel Anderson ${ }^{23}$ and Kondo Hamiltonian, 24.25 different twoimpurity clusters, $\stackrel{26,27,28,29,30,31,32}{2}$ and a host of related zero-dimensional problems. Recently, it was extended to equilibrium properties of impurity models with a bosonic bath $^{33.34}$ or even combinations of both fermionic and Bosonic baths 35

The Hamiltonian of a quantum impurity system is generally given by

$$
\mathcal{H}=\mathcal{H}_{\text {bath }}+\mathcal{H}_{\text {imp }}+\mathcal{H}_{\text {mix }},
$$

where $\mathcal{H}_{\text {bath }}$ models the continuous bath, $\mathcal{H}_{\text {imp }}$ represents the decoupled impurity, and $\mathcal{H}_{\text {mix }}$ describes the coupling between the two subsystems. Thermodynamic properties of such a quantum impurity system are very accurately obtained using the NRG. At the heart of this approach is a logarithmic discretization of the continuous bath, controlled by the discretization parameter $\Lambda>1,11$ the continuum limit is recovered for $\Lambda \rightarrow 1$. Using an appropriate unitary transformation, 11 the Hamiltonian is mapped onto a semi-infinite chain, with the impurity coupled to the open end. By construction, the $N^{\text {th }}$ site of this chain couples only to its immediate neighbors, which allows to write the Hamiltonian of the infinite chain as limit for $N \rightarrow \infty$ of a sequence of finite chains, denoted by $H_{N}$, with a unique prescription $H_{N} \mapsto H_{N+1}$, the RG equation of the NRG. The $N^{\text {th }}$ link along the chain represents an exponentially decreasing energy scale $D_{N} \sim \Lambda^{-N / 2}$ for a fermionic $\frac{11}{1}$ and $D_{N} \sim \Lambda^{-N}$ for a bosonic bath ${ }^{33}$ Using this hierarchy of scales, the sequence of finite-size Hamiltonians $\mathcal{H}_{N}$ for the $N$-site chain ${ }^{44}$ is solved iteratively, i.e. starting with $N=0$ one constructs the Hamiltonian $H_{0}$, diagonalizes it, adds the next site to obtain $H_{1}$, diagonalizes it etc. The problem of an exponentially increasing Hilbert space is resolved by observing, that, due to the exponential decrease of the energy scales, only lowenergy states actually contribute in the step $N \rightarrow N+1$; one thus discards the high-energy states before moving on to the next step to maintain a manageable number of states, we denote with $N_{S}$ in the following. The reduced basis set of $\mathcal{H}_{N}$ obtained that way is expected to faithfully describe the spectrum of the full Hamiltonian on a scale of the order of $D_{N}$, corresponding to a temperature $T_{N} \sim D_{N} \stackrel{11}{11}$ Note that even from this brief discussion it is evident that for a given step $N$ all information about energies $E \gg D_{N}$ has been lost, while no information about energy scales $E \ll D_{N}$ is available yet. Thus, to calculate dynamical quantities with a similar accuracy as thermodynamic, one has to tackle the problem of correctly mixing information from earlier and later NRG steps.

\section{A. Complete Basis Set}

In a recent extension of the NRG to real-time dynamics out of equilibrium ${ }^{20.21}$ a complete basis set for such a Wilson chain of length $N$ has been identified. Furthermore, this complete basis set also forms an approximate eigenbasis of the Hamiltonian $H_{N}$. Since this complete basis set plays a crucial role in the derivation of the analytical expression for spectral functions, we summarize briefly the main ideas 20 of the proof of completeness discussed extensively in Ref. 21.

At first sight the claim that the NRG automatically generates a complete basis set, which is also an approximate eigenbasis of the chain Hamiltonian $H_{N}$, might appear contradictory. A renormalization procedure is usually viewed as a clever way to identify the relevant degrees of freedom by reducing the dimensionally of the underlying Fock space. In order to excavate the optimally adapted complete basis set, we have to shift perspectives how to view the NRG algorithm.

There are two possible ways to interpret the iterative NRG solution of the $N$-site chain. In the traditional picture one starts from a core cluster that consists of the impurity degrees of freedom and the $N=0$ site, and enlarges the chain by one site at each NRG step. Alternatively, one can view the NRG procedure as starting from the full chain of length $N$, but with the hopping matrix elements set to zero along the chain. At each successive step another hopping matrix element is switched on, until the full Hamiltonian $\mathcal{H}_{N}$ is recovered. In this latter picture, to be adopted below, the entire sequence of Hamiltonians $\mathcal{H}_{m}$ with $m \leq N$ act on the Fock space of the $N$-site chain.

Accordingly, each NRG eigen-energy of $\mathcal{H}_{m}$ has an extra degeneracy of $d^{(N-m)}$, where $d$ is the number of distinct states at each site along the chain. The extra degeneracy stems from the $N-m$ "environment" sites at the end of the chain, depicted in Fig. 1]

The set of eigenstates of $\mathcal{H}_{m}$, conventionally denoted as $\{|r\rangle\}$, can be formally constructed from the complete basis set $\left\{\left|\alpha_{i m p}, \alpha_{0}, \cdots, \alpha_{N}\right\rangle\right\}$ of the NRG chain of length $N$ where the $\alpha_{i}$ label the configurations on each chain link $i$. Since $\mathcal{H}_{m}$ does not act on the chain links $m+1, \cdots, N$, $|r\rangle$ can be written as $|r, e ; m\rangle$ where the "environment" variable $e=\left\{\alpha_{m+1}, \cdots, \alpha_{N}\right\}$ encodes the $N-m$ site labels $\alpha_{m+1}, \cdots, \alpha_{N}$. The index $m$ is used in this notation to record where the chain is partitioned into a "subsys- 


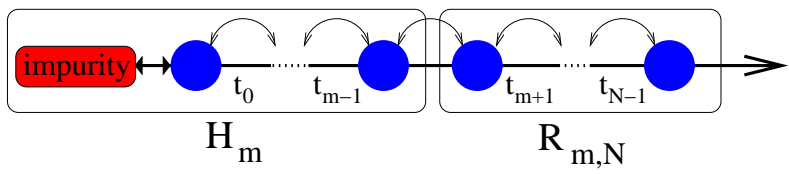

FIG. 1: (color online) The full Wilson chain of length $N$ is divided into a sub-chain of length $m$ and the "environment" $R_{m, N}$. The Hamiltonian $\mathcal{H}_{m}$ can be viewed either as acting only on the sub-chain of length $m$, or as acting on the full chain of length $N$, but with the hopping matrix elements $t_{m}, \cdots, t_{N-1}$ all set to zero. The former picture is the traditional one. In this paper we adopt the latter point of view.

tem" and an "environment" (see Fig. 1).

Consider now the first iteration $m_{\min }$ at which states are discarded. In order to keep track of the complete basis set of the $N$-site chain, we formally divide the eigenstates $|r, e ; m\rangle$ into two distinct subsets: the discarded high-energy states $\left\{|l, e ; m\rangle_{\text {dis }}\right\}$ and the retained low-energy states $\left\{|k, e ; m\rangle_{k p}\right\}$. In the course of the NRG, only the latter states are used to construct the next Hamilton matrix $\mathcal{H}_{m+1}$ within the reduced subspace $\left\{\left|k, \alpha_{m+1}, e^{\prime} ; m\right\rangle\right\}$. Note, however, that the sum of both subsets still constitutes a complete basis set for the $\mathrm{N}$ site chain. Repeating this procedure at each subsequent iteration, we recursively divide the retained subset into a discarded part and a retained part. Then, the collection of all discarded eigenstates $|l, e ; m\rangle_{d i s}$ together with the eigenstates of the final NRG iteration $N$ combine to form a complete basis set for the entire Fock space $\mathcal{F}_{N}$. Regarding all eigenstates of the final NRG iteration as discarded, one can formally write the Fock space of the $N$-site chain in the form $\mathcal{F}_{N}=\operatorname{span}\left\{|l, e ; m\rangle_{\text {dis }}\right\}$. Since all states are retained in the course of this construct, the following completeness relation obviously holds:

$$
\sum_{m=m_{\min }}^{N} \sum_{l, e}|l, e ; m\rangle_{\text {dis }}{ }_{\text {dis }}\langle l, e ; m|=1 .
$$

Here the summation over $m$ starts from the first iteration $m_{\min }$ at which a basis-set reduction is imposed. All traces below will be carried out with respect to this basis set. Hence, the evaluation of the spectral functions will not involve any truncation error. Note also that we made no reference to a particular Hamiltonian $\mathcal{H}$ in constructing the basis set $\left\{|l, e ; m\rangle_{\text {dis }}\right\}$.

Another useful identity to be used below pertains to the subspace retained at iteration $m,\left\{|k, e ; m\rangle_{k p}\right\}$. To this end, we note that the sum in Eq. (2) can always be divided into two complementary parts $1_{m}^{-}$and $1_{m}^{+}$:

$$
\begin{aligned}
1_{m}^{-} & =\sum_{m^{\prime}=m_{m i n}}^{m} \sum_{l^{\prime}, e^{\prime}}\left|l^{\prime}, e^{\prime} ; m^{\prime}\right\rangle_{\text {dis dis }}\left\langle l^{\prime}, e^{\prime} ; m^{\prime}\right|, \\
1_{m}^{+} & =\sum_{m^{\prime}=m+1}^{N} \sum_{l^{\prime}, e^{\prime}}\left|l^{\prime}, e^{\prime} ; m^{\prime}\right\rangle_{\text {dis dis }}\left\langle l^{\prime}, e^{\prime} ; m^{\prime}\right| . \\
& =\sum_{k, e}|k, e ; m\rangle_{k p k p}\langle k, e ; m| .
\end{aligned}
$$

with the completeness relation

$$
1=1_{m}^{-}+1_{m}^{+}
$$

Note that for $m=N$ only $1_{m}^{-}$exists.

\section{B. Definition of the Green's function}

The textbook definition of the retarded Green's function is given by

$$
G_{A, B}(t)=-i \Theta(t) \operatorname{Tr}\left[\hat{\rho}[A(t) B]_{-s}\right]
$$

where $[A, B]_{s}=A B-s B A$ with $s=1$ for bosonic operators $A, B$ and $s=-1$ for fermionic operators. In our investigation, we restrict ourselves to local operators $A$ and $B, 20$

As already mentioned, the fundamental philosophy of the NRG is that a chain of length $N$ corresponds to a temperature scale $T \sim D_{N}$, hence $\beta E_{l}^{m} \gg 1$ for all $m<$ $N$, where $E_{l}^{m}$ denotes the eigen energies of $\mathcal{H}_{m}$. In the $\mathrm{NRG}$, the thermodynamic density operator $\hat{\rho}$ is therefore represented only by the states of the last iteration $N$

$$
\begin{aligned}
\hat{\rho} & =\frac{1}{Z} e^{-\beta H} \approx \frac{1}{Z_{N}} \sum_{l} \rho_{l}|l ; N\rangle\langle l ; N| \\
\rho_{l} & =\frac{e^{-\beta E_{l}^{N}}}{Z_{N}}
\end{aligned}
$$

and $Z_{N}=\sum_{l} e^{-\beta E_{l}^{N}}$.

If we were able to solve the NRG chain of length $N$ without any truncation, the Green's function $G_{A, B}(z)$ would be given by the textbook Lehmann representation

$$
\begin{aligned}
G_{A, B}(z) & =\int_{0}^{\infty} d t e^{i z t} G_{A, B}(t) \\
& =\frac{1}{Z} \sum_{l, l^{\prime}} A_{l l^{\prime}} B_{l^{\prime} l} \frac{e^{-\beta E_{l}}-s e^{-\beta E_{l^{\prime}}}}{z+E_{l}-E_{l^{\prime}}}
\end{aligned}
$$

\section{Derivation of the NRG Green's function}

Let us start from the retarded Green's function looking only at the first term from the commutator and insert two completeness relations (5). We then obtain 


$$
\begin{aligned}
\operatorname{Tr}\left[\hat{\rho} e^{i H t} A e^{-i H t} B\right]= & \sum_{l, e, m} \sum_{l^{\prime}, e^{\prime}, m^{\prime}} \operatorname{Tr}\left[\hat{\rho} e^{i H t}|l, e ; m\rangle\left\langle l, e ; m\left|A e^{-i H t}\right| l^{\prime}, e^{\prime} ; m^{\prime}\right\rangle\left\langle l^{\prime}, e^{\prime} ; m^{\prime}\right| B\right] \\
= & \sum_{m} \sum_{l, e} \sum_{l^{\prime}, e^{\prime}} \text { dis }\left\langle l, e ; m\left|A e^{-i H t}\right| l^{\prime}, e^{\prime} ; m\right\rangle_{\text {dis dis }}\left\langle l^{\prime}, e^{\prime} ; m\left|B \hat{\rho} e^{i H t}\right| l, e ; m\right\rangle_{\text {dis }} \\
& +\sum_{m} \sum_{l, e} \sum_{k, e^{\prime}} \text { dis }\left\langle l, e ; m\left|A e^{-i H t}\right| k, e^{\prime} ; m\right\rangle_{k p} k p\left\langle k, e^{\prime} ; m\left|B \hat{\rho} e^{i H t}\right| l, e ; m\right\rangle_{\text {dis }} \\
& +\sum_{m} \sum_{l, e^{\prime}} \sum_{k, e} \text { dis }\left\langle l, e^{\prime} ; m\left|B e^{i H t} \hat{\rho}\right| k, e ; m\right\rangle_{k p k p}\left\langle k, e ; m\left|A e^{-i H t}\right| l, e^{\prime} ; m\right\rangle_{d i s} .
\end{aligned}
$$

For the second term from the commutator in (6) one obtains a similar expression. Here and in the following we use the convention that an index $l$ labels a discarded state, while an index $k$ represents a state kept at a certain chain length $m$. The first term in (10) arises from $m^{\prime}=$ $m$, the second from $m^{\prime}>m$ and the third from $m^{\prime}<$ $m$, where we made use of Eq. (4). This trick ${ }^{20}$ allows to express the Green's function as sum over equal shell contributions only. Note that this is an exact formula: no approximations have been made so far!

Since the state $|s, e ; m\rangle$ is an eigenstate of $H_{m}$, i.e. $H_{m}|s, e ; m\rangle=E_{s}^{m}|s, e ; m\rangle$, we will now use the approximation $H|s, e ; m\rangle \approx E_{s}^{m}|s, e ; m\rangle$. This approximation, justified due to the energy hierarchy implied by the logarithmic discretization,, 45 is completely in the spirit of the NRG and in fact used in the calculation of thermodynamics properties. ${ }^{11}$ Note, that this will be the only approximation made, which is of energetic nature and unrelated to any truncation error. We thus eval- uate $\exp (i H t)|s, e ; m\rangle \approx \exp \left(i E_{s}^{m} t\right)|s, e ; m\rangle$ and Laplace transform all contributions. The first term from (10) and the corresponding expression for the second term from the commutator contains only discarded states and reduces, therefore, to the last iteration $m=N$ due to the representation of the density operator in NRG by Eq. (7). It yields as contribution to $G_{A, B}(z)$

$$
\begin{aligned}
G_{A, B}^{i}(z)= & \frac{1}{Z} \sum_{l, l^{\prime}}\left\langle l ; N|A| l^{\prime} ; N\right\rangle\left\langle l^{\prime} ; N|B| l ; N\right\rangle \\
& \times \frac{e^{-\beta E_{l}^{N}}-s e^{-\beta E_{l^{\prime}}^{N}}}{z+E_{l}^{N}-E_{l^{\prime}}^{N}}
\end{aligned}
$$

In the next two terms the summation over all energy shells has to be evaluated, because the states $|k, e ; m\rangle_{k p}$ are not orthogonal to $|l ; N\rangle$. We obtain, using $\hat{\rho}|l, e ; m\rangle_{\text {dis }}=0$ for $m<N, 21$

$$
G_{A, B}^{i i}(z)=\sum_{m=m_{m i n}}^{N-1} \sum_{l, e} \sum_{k, e^{\prime}}\left\langle l, e ; m|A \hat{\rho}| k, e^{\prime} ; m\right\rangle\left\langle k, e^{\prime} ; m|B| l, e ; m\right\rangle \frac{-s}{z+E_{l}^{m}-E_{k}^{m}}
$$

and

$$
G_{A, B}^{i i i}(z)=\sum_{m=m_{m i n}}^{N-1} \sum_{l, e^{\prime}} \sum_{k, e}\left\langle l, e^{\prime} ; m|B \hat{\rho}| k, e ; m\right\rangle\left\langle k, e ; m|A| l, e^{\prime} ; m\right\rangle \frac{1}{z+E_{k}^{m}-E_{l}^{m}}
$$

Since in the last iteration there are no kept states, all are considered discarded, $m=N$ does not contribute to $G^{i i}$ and $G^{i i i}$. Now we insert a completeness relation $\left(1_{m}^{+}+1_{m}^{-}\right)$between $A$ and the density operator in $G_{A, B}^{i i}(z)$ and $B$ and the density operator in $G_{A, B}^{i i i}(z)$ and make use of $1_{m}^{-} \hat{\rho}=0$ for all $m<N^{21}$ Because $A$ and $B$ are local operators, 46 the matrix elements

$$
\left\langle k, e ; m|A| l, e^{\prime} ; m\right\rangle=s^{n_{e^{\prime}}} \delta_{e, e^{\prime}} A_{k, l}(m)
$$

are diagonal and independent of the environment variables $e . n_{e^{\prime}}$ denotes the number of Fermions in the environment times the total number of Fermions created by $\hat{A}$. Since the matrix elements of $A$ and $B$ are evaluated simultaneously, the total phase factor is given by $\left[s^{n_{e^{\prime}}}\right]^{2}=1$ for operator $A$ and $B^{\dagger}$ imposing the same change of the total particle number. Therefore, the trace over the environment only acts on the density operator 
$\hat{\rho}$ and the final result

$$
\begin{aligned}
G_{A, B}^{i i}(z)= & \sum_{m=m_{m i n}}^{N-1} \sum_{l} \sum_{k, k^{\prime}} A_{l, k^{\prime}}(m) \rho_{k^{\prime}, k}^{\mathrm{red}}(m) B_{k, l}(m) \\
& \times \frac{-s}{z+E_{l}-E_{k}}
\end{aligned}
$$

and

$$
\begin{aligned}
G_{A, B}^{i i i}(z)= & \sum_{m=m_{m i n}}^{N-1} \sum_{l} \sum_{k, k^{\prime}} B_{l, k^{\prime}}(m) \rho_{k^{\prime}, k}^{\mathrm{red}}(m) A_{k, l}(m) \\
& \times \frac{1}{z+E_{k}-E_{l}} \\
G_{A, B}(z)= & G_{A, B}^{i}(z)+G_{A, B}^{i i}(z)+G_{A, B}^{i i i}(z)
\end{aligned}
$$

is formulated in terms of the reduced density matrix ${ }^{36.37}$

$$
\rho_{k, k^{\prime}}^{\mathrm{red}}(m)=\sum_{e}\left\langle k, e ; m|\hat{\rho}| k^{\prime}, e ; m\right\rangle,
$$

first introduced to the calculations of NRG spectral functions by Hofstetter ${ }^{16}$ The part $G^{i i}(z)$ describes negative frequency excitations, while $G^{i i i}(z)$ accounts for positive frequency excitations for all $m<N$ because $E_{l}-E_{k}>0$ by construction. $G^{i}(z)$ sums all excitations of the last iteration $N$ and has the form of the usual Lehmann representation Eq. (9).

A few words are in place to pinpoint the difference to the Hofstetter approach ${ }^{16}$ to calculate spectral functions with NRG. Although our reduced density matrix $\rho^{\text {red }}$ is identical to the one given in Eq. (7) in Ref. 16, our rigorous derivation differs in the summation of excitations contributing to the Green's function. Eqs. (15) and (16) state clearly that one must only include excitations between a discarded and kept state while in the Hofstetter approach $^{16}$ the summation index $l$ runs over all states present at iteration $m$. This leads to an overcounting of contributions, by the way inherent to all previous approaches to NRG spectral functions, see for details Refs. 15 16 17 1819 and references therein. The origin of the restriction of summation in Eqs. (15) and (15) is quite obvious: All kept states in iteration $m$ span the Fock-space of Hamiltonian $H_{m+1}$ and, therefore, will contribute to the excitations at a latter iteration $m^{\prime}>m$. They must not be included at iteration $m$.

In addition, the phenomenological patching algorithms $\stackrel{15}{\stackrel{15}{1}}$ where spectral information from different energy shells are "merged" become obsolete in our approach. Equations (11:16) state exactly which excitations contribute at which Wilson shell. Obeying this summation restriction ensures the fulfillment of the spectral sum rules independent of the number $N_{S}$ of NRG states kept at each iteration. With a little algebra and the completeness relations (5), we show in appendix
A that the spectral sum rule

$$
\begin{aligned}
C & =\oint \frac{d z}{2 \pi i} G_{A, B}(z) \\
& \left.=\sum_{\alpha=i, i i, i i i} \oint \frac{d z}{2 \pi i} G_{A, B}^{\alpha}(z)=\operatorname{Tr}\left[\rho[A, B]_{s}\right]\right)
\end{aligned}
$$

is always fulfilled exactly.

Therefore, the spectral function will be correctly normalized independent of the number of states $N_{S}$ kept after each NRG iteration. Note that the NRG truncation only influences the partitioning of the states, but never the completeness of the basis. Hence, the spectral functions become more robust to truncation errors, as will be shown in section [II by using an extremely low number of kept NRG states. The spectral sum rule is a consequence of an operator identity and independent of approximations made in the dynamics as long as no states are discarded in the calculation.

\section{Occupation number}

Let us specialize to the local spin-dependent fermionic spectral function, i.e. $A=f_{\sigma}$ and $B=f_{\sigma}^{\dagger}$. The local occupation $\left\langle f_{\sigma}^{\dagger} f_{\sigma}\right\rangle$ can be expressed with the spectral integral as

$$
\begin{aligned}
\left\langle f_{\sigma}^{\dagger} f_{\sigma}\right\rangle & =\int_{-\infty}^{\infty} \frac{d \omega}{\pi} f(\omega) \Im m G_{f_{\sigma}, f_{\sigma}^{\dagger}}(\omega-i \delta) \\
& =\oint \frac{d z}{2 \pi i} f(z) G_{f_{\sigma}, f_{\sigma}^{\dagger}}(z)
\end{aligned}
$$

where $f(\omega)$ is the Fermi function. By substituting Eqs. (1116) for $G_{f_{\sigma}, f_{\sigma}^{\ddagger}}(z)$ and evaluating the contour integration, we show in appendix B explicitly that our expressions approximately - at $T=0$ even exactly - reproduce the expectation value $\left\langle f_{\sigma}^{\dagger} f_{\sigma}\right\rangle$ calculated directly with the NRG. In contrast to the spectral sum rule, however, which is exact and, therefore, reproduced in our numerics with machine accuracy, the accuracy of the occupation numbers calculated from the spectra depends on the validity of the assumption of vanishing Boltzmann factors. Therefore, we expect it to show a certain error at finite temperatures. Nevertheless, we find that the deviation between the NRG values and the ones obtained by the numeric evaluation ${ }^{47}$ of (20) remains less than $10^{-4}$. This implies also that the NRG value for quantities like the local magnetization $m=\sum_{\sigma} \sigma\left\langle f_{\sigma}^{\dagger} f_{\sigma}\right\rangle / 2$ is accurately reproduced by our formulation of the Green's function. This is a significant improvement over the Hofstetter approach, where deviations on the percent level between $m^{N R G}$ and $m^{G F}$ have been reported. ${ }^{16}$ 


\section{RESULTS}

\section{A. Single impurity Anderson model}

The general scheme for the calculation of spectral functions presented in section III not make any reference to a certain model or bath statistics. Therefore, our algorithm is suitable for any quantum impurity system. In order to demonstrate the virtue of the new approach, we will present in this section as an important example calculations for the spin-dependent single-particle spectral functions of the single impurity Anderson model (SIAM) with and without an external magnetic field at $T=0$ and finite $T$.

The Hamiltonian of the SIAM ${ }^{6,12,13}$

$$
\begin{aligned}
\mathcal{H}= & \sum_{k \sigma} \epsilon_{k \sigma} c_{k \sigma}^{\dagger} c_{k \sigma}+\sum_{\sigma}\left(\epsilon_{f}-\sigma H\right) f_{\sigma}^{\dagger} f_{\sigma} \\
& +\frac{U}{2} \sum_{\sigma} n_{\sigma}^{f} n_{-\sigma}^{f}+V \sum_{k \sigma}\left(c_{k \sigma}^{\dagger} f_{\sigma}+f_{\sigma}^{\dagger} c_{k \sigma}\right)
\end{aligned}
$$

consists of a single local state, which we will denote with $f$, with energy $\epsilon_{f}$ and Coulomb repulsion $U$, coupled to a bath of conduction electrons with creation operators $c_{k \sigma}^{\dagger}$ and energies $\epsilon_{k \sigma}$. The local level is subject to a Zeemann splitting in an external magnetic field $B$. Note that we denote with $H=g \mu_{\mathrm{B}} B / 2$ the Zeeman energy and the total splitting $\left|\epsilon_{\uparrow}-\epsilon_{\downarrow}\right|=2 H$. We employ Wilson's $\mathrm{NRG}^{12,13}$ to generate the eigen-energies, the matrix elements and basis set needed for the Green's function.

So far, all analytical calculations were performed using the discrete NRG spectrum. To obtain a continuous spectral function from the set of discrete $\delta$-functions occurring in $G_{A, B}(z)$, we have to introduce a coarse-graining. Due to the exponentially decreasing energy scales, a broadening on a logarithmic mesh by a Gaussian

$$
\delta\left(\omega-\omega_{n}\right) \rightarrow \frac{e^{-b^{2} / 4}}{b \omega_{n} \sqrt{\pi}} \exp \left\{-\left(\frac{\ln \left(\omega / \omega_{n}\right)}{b}\right)^{2}\right\}
$$

is typically used. ${ }^{17.38}$ Since this broadening function is properly normalized to one, the spectral weight is conserved and no principle inaccuracies are introduced by this procedure.

\section{B. Spectral functions for $T=0$}

In the following, we specialize to $A=f_{\sigma}$ and $B=f_{\sigma}^{\dagger}$. For comparison we calculated the raw NRG spectral function in three different ways: (i) by the conventional way as described in Refs. 15 17 labelled as (CON) in the following, (ii) by the Hofstetter approach ${ }^{16}$ (HA) and by our method defined by Eqs. (11)-(17) labelled complete Fock space approach (CFS). As long as not stated otherwise, all energies are measured in units of the half band width $D$, and for simplicity only a symmetric conduction band is considered with a constant density of states $\rho_{0}=1 /(2 D) \Theta(D-|\omega|), 11$

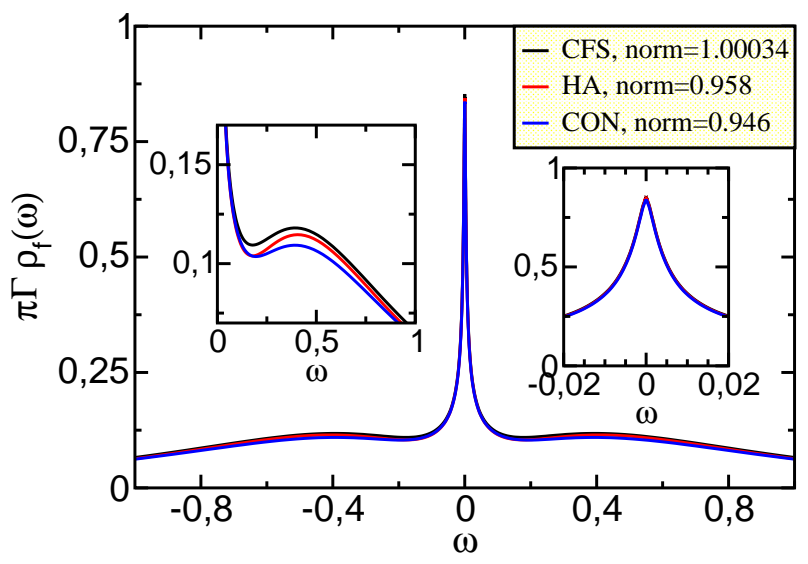

FIG. 2: (color online) Comparison of the spectral function for the three different methods for the symmetric case $\epsilon_{f} / D=$ $-U / D 2=0.5$. The inset on the left side emphasizes the broad charge excitation at $\epsilon_{f}$, the inset on the right side zooms in at the Abrikosov-Suhl resonance (ASR). NRG parameters: $\Gamma / D=\pi V^{2} \rho_{0} / D=0.1, \Lambda=2.5, N_{S}=500, b=0.8, T=0$

In Fig 2 we show a comparison of spectral functions calculated by the three different methods using identical NRG input data and broadening parameters. All three methods agree on the shape and the height of the manybody resonance (ASR) at the Fermi energy and all methods do not reach the unitary limit of $\pi \Gamma \rho(0)=1$ as predicted by the density of states rule 39 The spectral functions, however, differ in their high energy features. The conventional spectral function underestimates the charge excitations and only reaches a total spectral weight of $C=0.946$. The Hofstetter approach ${ }^{16}$ yields a somewhat improved value of $C=0.956$ because the high energy part of its spectrum, which carries most of the spectral weight, lies between our and the conventional curve. The spectral function based upon our new approach, however, reaches the spectral weight $C=1.00034$. It deviates from the exact value of $C=1$ only by the error introduced by the numerical $\omega$ integration. To verify this, we add all spectral weights from Eqs. (11)-(16) directly and obtain $|C-1|=10^{-12} \ldots 10^{-15}$, i.e. the norm is $C=1$ within machine accuracy $\stackrel{48}{ }$ For this symmetric case, its obvious that our approach also yields the exact occupation number of $n_{\sigma}^{f}=\left\langle\hat{n}_{\sigma}^{f}\right\rangle=0.5$ for each spin direction while the other two approaches have a $5.7 \%(\mathrm{CON})$ or $4.6 \%(\mathrm{HA})$ error. Note, however, that the latter two still give the correct value $C / 2$ with respect to their respective norm.

At this point a comment on the violation of the density of states rule $\pi \Gamma \rho(0)=1$ for $T \rightarrow 0$ seems in order. Since the NRG itself does only provide the weight of $\delta$-peaks, the height of the coarse-grained spectrum will depend on the density of these peaks, i.e. the number of states available in the calculation, and the choosen broadening function. This does not imply the violation of Friedel 
sum rule ${ }^{39}$, too, which relates the scattering phases to the number of displaced electrons in each spin channel. Since the NRG fixed point spectrum contains the correct scattering phases, it is accurately fulfilled. However, the relation between the phase shift and the local spectral function is in general not very accurately reproduced due to the aforementioned reasons. Nevertheless, the density of states sum rule can be satisfied with an error of less than $3 \%$ using small values of $\Lambda$, more NRG states and a smaller broadening ${ }^{17}$ as used in Fig 2

To our knowledge, only the indirect calculation of the physical Green's function by expressing its self-energy by the ratio of two correlation functions 18 yields values which are accurate enough to reproduce the correct height of $\rho(0, T=0)$ nearly independent of the broadening parameters. In particular, this is of importance to prove the pinning of the spectral function for the twochannel Anderson model at half the unitary limit.23

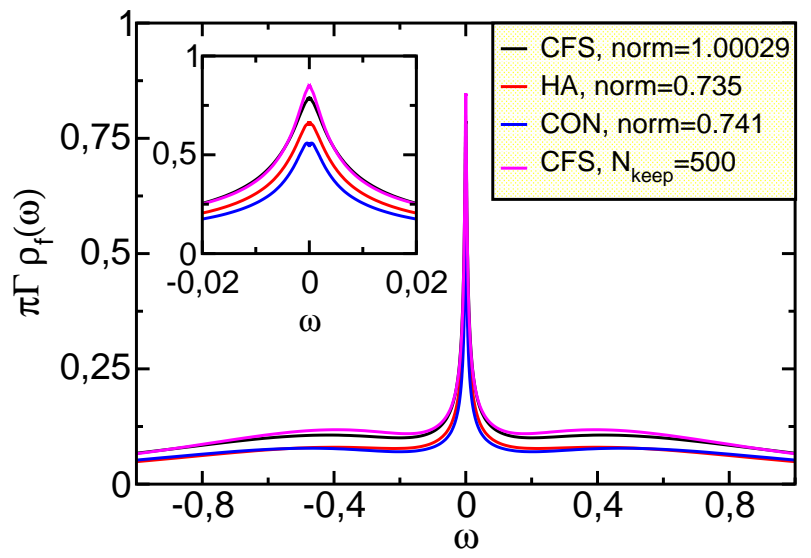

FIG. 3: (color online) Comparison of the spectral function for the three different methods for the same model and NRG parameters as in Fig. 2 but with only $N_{S}=50$ states kept in each NRG iteration. The inset zooms in onto the ASR.

We have put our method to an extreme test by reducing the number of states $N_{S}$ kept after each NRG step to $N_{S}=50$ for the same $\Lambda=2.5$ as before. This is definitely stretching the limit of the conventional NRG beyond its accuracy. The results for the spectral functions are plotted in Fig. 3. Only data for frequency $|\omega|>10^{-7}$ are included, which, however, is much smaller than the Kondo temperature $T_{K}$ as estimated from the width of the ASR. The inaccuracy of the matrix elements for extremely small frequencies $|\omega|<10^{-7}$ yields erratic results in this interval which, therefore, are omitted here. While the conventional and the HA methods significantly loose spectral weight, the spectral weight of CFS spectral function remains at the exact value $C=1$. Since the discretization of the energy mesh was the same in all figures, the deviation from the sum rule of $3 \times 10^{-4}$ again stems only from the numerical integration. For comparison we also added the CFS curve from Fig. 2 calculated with $N_{S}=500$ NRG states. Both CFS curves agree extremely well. In particular, the weight under the charge peak is only slightly redistributed and the shape of the ASR remains unaltered. Only at very low frequency, the inaccuracy of the underlying NRG input data takes its toll: the ASR peak height is further reduced. The other two methods, however, exhibit strong dependence on $N_{S}$ in all energy regimes. We must report an error in the spectral weight and the occupancy $n_{\sigma}^{f}$ of $\approx 35 \%$ for both the conventional and the HA method. In all cases the same broadening parameter $b=0.8$ was used.

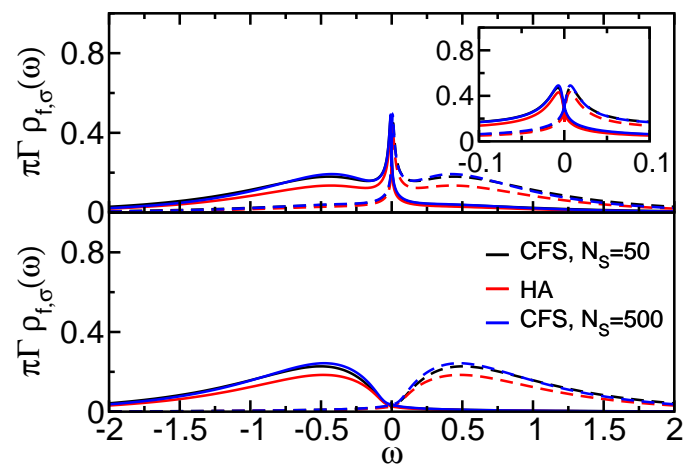

FIG. 4: (color online) Comparison of the spectral function obtained from CFS and HA approaches in a finite magnetic field (a) $H=0.005$ and (b) $H=0.1$. The full curves represent spin up, the dashed ones spin down. The inset in (a) is a zoom into the region around the Fermi level showing the splitting and suppression of the Kondo resonance. Model and NRG parameters as in Fig. 3. The norm and occupancies obtained are compared in Tab. I

While the conventional method yields quite reliable spectral information for small $\Lambda$ and large number of states in the absence of a magnetic field, it fails for finite magnetic field, where the fixed point for $T \rightarrow 0$ yields a complete redistribution of spectral weight on all energy scales compared to the spectral function ${ }^{16}$ at $H=0$. Therefore, it is omitted in Fig. 4. where we compare spectral functions for two magnetic fields, $H=0.005 \approx T_{K}$ (Fig. 4. and $H=0.1 \gg T_{K}$ (Fig. 4b); the results for the majority spin are plotted as solid lines, the ones for the minority spin as dashed lines. For $H \approx T_{K}$ the ASR is split and already reduced to half its original height, while for $H \gg T_{K}$ it has vanished completely, as expected. Again, we display the data for $N_{S}=50$ to highlight the differences in the methods. While our method and the HA approach exhibit the same overall features, i.e. splitting and reduction respectively complete lack of the ASR and a major redistribution of spectral weight, the charge excitation peak contains much more spectral weight in the CFS as the HA curve. As before, the CFS spectral function fulfills the spectral sum rule with the accuracy of $10^{-4}$ while we note a $20 \%$ error in the HA curve for these extreme set of parameters. The CFS results for $N_{S}=500$ given by the blue curves in Fig. 4 by and large again lie on top of the CFS curves for $N_{S}=50$, once more emphasizing that within the CFS neither the accuracy of the occupancy nor the one of the spectral 


\begin{tabular}{llrrrrr} 
& Method & Norm & \multicolumn{2}{c}{$\left\langle n_{\sigma}\right\rangle_{G F}$} & \multicolumn{2}{c}{$\left\langle n_{\sigma}\right\rangle_{N R G}$} \\
& & & $\sigma=\uparrow$ & $\sigma=\downarrow$ & $\sigma=\uparrow$ & $\sigma=\downarrow$ \\
\hline (a) & CFS(500) & 1 & 0.807 & 0.193 & 0.807 & 0.193 \\
$H=0.005$ & CFS(50) & 1 & 0.806 & 0.194 & 0.806 & 0.194 \\
& HA & 0.747 & 0.600 & 0.147 & 0.806 & 0.194 \\
\hline (b) & CFS(500) & 1 & 0.953 & 0.047 & 0.953 & 0.047 \\
$H=0.1$ & CFS(50) & 1 & 0.953 & 0.047 & 0.953 & 0.047 \\
& HA & 0.793 & 0.755 & 0.038 & 0.953 & 0.047 \\
\hline
\end{tabular}

TABLE I: Norm and occupancies for the calculations with finite magnetic field in Fig. 4 The last columns contain the occupancies obtained from the thermodynamic expectation values.

functions does critically depend on the number of states kept.

This feature becomes even more apparent from the actual numbers for the norm and the spin-dependent occupation numbers listed in Tab. I The last column shows for comparison the occupancies for the two spin directions as calculated directly from the NRG level flow 11 Note that the difference in the occupancies between $N_{S}=500$ and $N_{S}=50$ in the CFS appear in the thermodynamic occupation numbers, too. On the other hand, there exist significant differences in the magnetization $m=\left(n_{\uparrow}-n_{\downarrow}\right) / 2$ obtained from the HA method, which yields a $30 \%$ error compared to the reference NRG magnetization and our CFS method.

We like to emphasize that the rather large error of the HA method results from the unusual low number of NRG state $N_{S}$ kept after each NRG iteration. Increasing the number of states and reducing $\Lambda$ one can increase the accuracy of the HA method considerably, but typically is always left with about $4 \%$ error according to Tab. I in Ref. 16. Our motivation for choosing $N_{S}=50$ was to put our initial claim to an extreme test: The spectral sum rule and the occupation numbers are reproduced with high accuracy independent of the number $N_{S}$ of states kept after each iteration. Our equations (11)- 116) do not contain any truncation errors since we use a complete basis set. The errors in the CFS spectral functions are of purely energetic nature and will indeed be found in the thermodynamic quantities, too. As a consequence, the spectral functions appear to be largely insensitive to the number of NRG state kept.

We made the same observation as discussed above in the asymmetric case shown in Fig. 5(a). The overall shape of the spectral functions of all three methods agree pretty well. Again, we note differences in the deviations from the sum rules, see Tab. II While our new CFS method obeys the spectral weight sum rules and the occupation sum rule very accurately, the comparison of the actual numbers for norm and occupancy in Tab. III a) shows that the conventional and the HA method yields $C=0.974$ and the Green's function occupancy of $n_{\sigma}^{G F}=0.645$ deviates from the NRG occupancy $n_{\sigma}^{N R G}=0.666$ by $3 \%$.

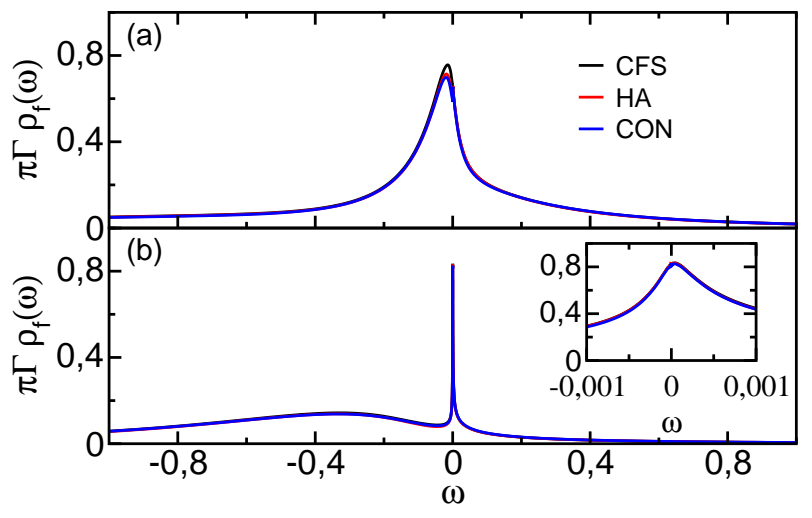

FIG. 5: (color online) Comparison of the spectral function for the three different methods for the asymmetric case (a) $U / D=1$ and $\epsilon_{f} / D=-0.9$, and (b) $U / D=1000$ and $\epsilon_{f} / D=-0.4$. NRG parameters: $\Gamma / D=\pi V^{2} \rho_{0} / D=$ $0.1, \Lambda=2.5, N_{S}=500$. The results for norm and occupancies obtained are collected in Tab. II]

\begin{tabular}{llrrr} 
& Method & Norm & $\langle n\rangle_{G F}$ & $\langle n\rangle_{N R G}$ \\
\hline (a) & CFS & 1 & 0.666 & 0.666 \\
$U=1$ & HA & 0.974 & 0.645 & 0.666 \\
& CON & 0.975 & 0.645 & 0.666 \\
\hline (b) & CFS & 0.543 & 0.915 & 0.915 \\
$U=\infty$ & HA & 0.523 & 0.878 & 0.915 \\
& CON & 0.534 & 0.893 & 0.915 \\
\hline
\end{tabular}

TABLE II: Norm and occupancies obtained with the different methods to calculate spectra for the asymmetric SIAM (see Fig. 5 for the parameters used). The last column shows the occupancy as obtained from the thermodynamic expectation value.

In Fig. 5. (b) the spectral functions for $U / D=1000$ are plotted. This corresponds to the limit $U \rightarrow \infty$ of the SIAM, i.e. the charge excitation between the singly and the doubly occupied state is effectively shifted to infinity. Therefore, the total spectral weight is reduced to $C=1-\left\langle\hat{n}_{f}\right\rangle / 2$, if we neglect the $\delta$-like peak at $\omega \approx U$. Nevertheless, adding all discrete NRG spectral weights yields $|C-1| \approx 10^{-12}-10^{-15}$ even in this case. Again, the values in Tab. III (b) show that the CFS spectral function agrees excellently with the exact norm but show a slight error of $0.03 \%$ in the occupancy, which we again attribute to the numerical integration, while the conventional and the HA methods have errors at least two orders of magnitude larger of about $4 \%$.

\section{Finite temperature spectral functions}

Let us now turn to the discussion of the temperature dependence of the spectral functions calculated with CFS method. Here, an additional problem arises, because the temperature scale in NRG is defined by the length $N$ of the chain. More precisely, a given chain length $N$ cor- 


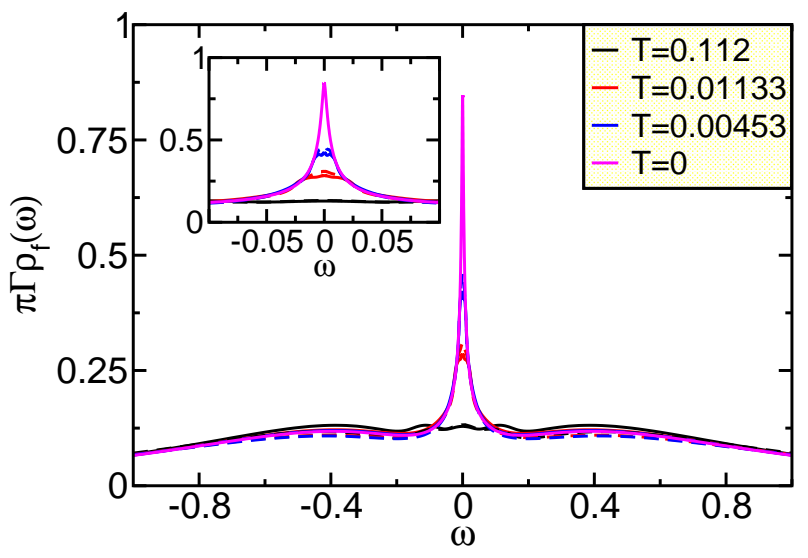

FIG. 6: (color online) Spectral function for temperatures $T=$ $0,0.00453 \approx T_{K}, 0.01133$ and $0.112 \gg T_{K}$ calculated with CFS. The full curves result from calculations with $N_{S}=500$, the dashed ones from calculations with $N_{S}=50$. Model and NRG parameters are otherwise the same as in Fig. 2

responds to a temperature $T_{N} \sim \Lambda^{-(N-1) / 2}$. Thus, a continuous variation of $T$ is not possible, only a discrete set of logarithmically varying temperatures is accessible. More problematic, however, is that due to the termination of the NRG iterations at chain length $N$ no information are available for excitation energies smaller than $T_{N}$, too 15 This means, that the part of the spectrum with $|\omega|<T_{N}$ is inaccurate in the sense that excitations on these scales cannot be taken into account properly. Furthermore, the limited information available for this energy range stems from all NRG iterations, and hence a Gaussian broadening (22) suitable for excitations collected from a single iteration cannot be used here. Instead, a Lorentzian broadening 15

$$
\delta\left(\omega-\omega_{n}\right) \rightarrow \frac{1}{2 \pi} \frac{\tilde{b}}{\left(\omega-\omega_{n}\right)^{2}+\tilde{b}^{2}}
$$

is used for $|\omega|<\alpha T_{N}$, where $\alpha$ sets the energy scale down to which we trust the NRG results $\frac{49}{4}$ Moreover, as discussed in detail in the appendix, the CFS still ensures a complete basis set and hence an exact norm for the spectral functions, but quantities obtained from spectral averages like occupancies must be expected to be less accurate than for $T=0$.

The development of the spectral function for $\Gamma / D=$ $\pi V^{2} \rho / D=0.1, \epsilon_{f}=-U / 2=-0.5 D$ as function of temperature can be found in Fig. [6] Even though we restrict the results presented in this section to the particlehole symmetric limit, all observations also hold for the asymmetric case with and without magnetic field. As expected, with increasing temperature the ASR is reduced, for $T \approx T_{K}$ to roughly half its original height. For $T \gg T_{K}$ it has completely vanished, leaving only the Hubbard peaks in the spectrum. For all spectra we find the norm to be exactly one as before and, due to particle-hole symmetry, the occupancy has to be onehalf. The full curves in Fig. 6] are results of NRG cal- culations with $N_{S}=500$ states, the dashed ones with $N_{S}=50$ states. As before, we can notice mild deviations of the spectra in the region of the Hubbard peaks and at very low energies, but the overall agreement of the spectra is quite good, thus again underlining the previous claim that spectra calculated with the CFS are rather insensitive to the number $N_{S}$ of states kept in each NRG iteration. Note that the deviations for $\omega \rightarrow 0$ can be easily accounted for by the fact that with varying $N_{S}$ the distribution of excitation energies in the NRG will differ, thus yielding a slightly different distribution of spectral weight due to the broadening (23).

The calculations for finite magnetic field $H=0.005$ are collected in Fig. [7 Here we only present results for

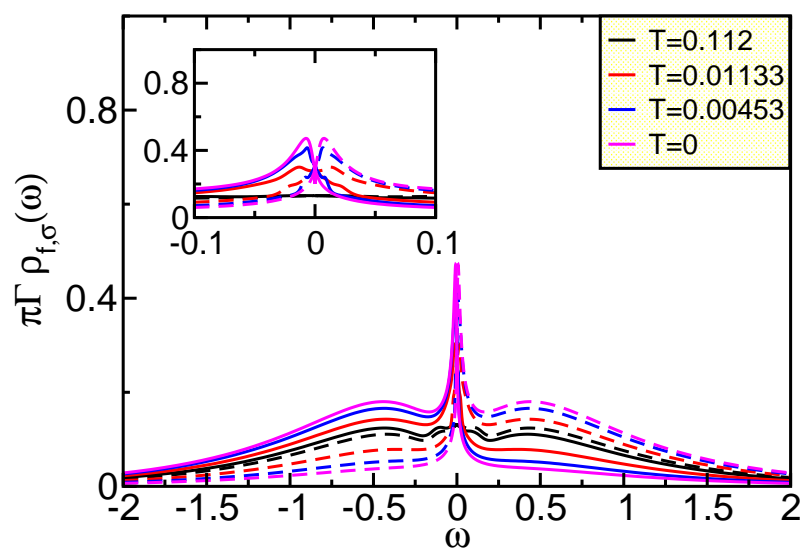

FIG. 7: (color online) Spectral function for temperatures $T=$ $0,0.00453 \approx T_{K}, 0.01133$ and $0.112 \gg T_{K}$ and finite magnetic field $H=0.005$ calculated with CFS. The full curves show the spectral function for spin up, the dashed curves spin down. Model and NRG parameters are otherwise the same as in Fig. 3 The values for norm and occupancies are listed in Tab. III

$N_{S}=50$, because as noted before the spectra for $N_{S}=$ 500 do not differ significantly. As in Fig. 3 we plotted the spectra for the majority spin as full curves, the ones for the minority spin as dashed curves. Again, the norm is exactly one in all cases. The occupancies obtained from integrating the spectra multiplied with the Fermi function at the appropriate temperature are listed in Tab. III for $N_{S}=500$ and $N_{S}=50$. To avoid additional inaccuracies introduced by the broadening (23) we have listed in Tab. III the sum of the weights of the raw NRG spectra multiplied with the Fermi function. As for $T=0$, the deviations to the thermodynamic values stay always less than $0.01 \%$. Numerical integration of the broadened spectra, on the other hand, will lead to stronger deviations, in particular for higher temperatures. For example, we find $\left\langle n_{\uparrow}\right\rangle_{G F}=0.516$ for $T=0.112$ and $N_{S}=500$, i.e. an error in the percent range. The value of $\int \rho(\omega) f(\omega)$ with $\rho(\omega)$ smoothened by the described combination of Gaussian and Lorentzian broadening deviates from the sum over the spectral weights of the poles of the discrete spectrum times the Fermi function at the 


\begin{tabular}{rrrrrrr}
$N_{S}$ & \multicolumn{1}{c}{$T$} & Norm & \multicolumn{2}{c}{$\left\langle n_{\sigma}\right\rangle_{G F}$} & \multicolumn{2}{c}{$\left\langle n_{\sigma}\right\rangle_{N R G}$} \\
& & & $\sigma=\uparrow$ & $\sigma=\downarrow$ & $\sigma=\uparrow$ & $\sigma=\downarrow$ \\
\hline 500 & 0 & 1 & 0.806 & 0.194 & 0.806 & 0.194 \\
50 & 0 & 1 & 0.807 & 0.193 & 0.807 & 0.193 \\
500 & 0.00453 & 1 & 0.723 & 0.277 & 0.723 & 0.277 \\
50 & 0.00453 & 1 & 0.729 & 0.271 & 0.729 & 0.271 \\
500 & 0.01133 & 1 & 0.629 & 0.371 & 0.629 & 0.371 \\
50 & 0.01133 & 1 & 0.635 & 0.365 & 0.635 & 0.365 \\
500 & 0.112 & 1 & 0.518 & 0.482 & 0.518 & 0.482 \\
50 & 0.112 & 1 & 0.519 & 0.481 & 0.519 & 0.481 \\
\hline
\end{tabular}

TABLE III: Norm and occupancies for the calculations with finite magnetic field and $T>0$ in Fig. 7 In addition the occupancies for $N_{S}=500$ are included in the table. The last columns contain the occupancies obtained from the thermodynamic expectation values.

pole energy, since the Lorentzian part extends in an uncontrolled manner to positive and negative frequencies. This systematic error, however, is still small even in these extreme cases.

\section{CONCLUSION}

We presented a novel approach for the calculation of local Green's functions for quantum impurity systems using Wilson's numerical renormalization group. Since it is based on the usage of a complete basis set for the Wilson NRG chain, recently introduced in the context of the time-dependent $\mathrm{NRG}^{20.21}$ quantities written as spectral sum rules are always exactly fulfilled, regardless of the size $N_{S}$ of the Hilbert space kept in each NRG iteration. Moreover, spectral averages like occupation numbers etc. are at least reproduced with unprecedented accuracy. We have demonstrated the quality of the approach by presenting results for the fermionic single-particle spectral function $\rho_{f}(\omega)$ of the single impurity Anderson model. We have shown explicitly the validity of our claim, that the resulting spectral functions are very insensitive to the number of NRG states kept in the iteration. This is a consequence of using a complete basis set in the derivation of the Lehmann representation. Equations (11)-(16) precisely account for which excitation contributes at which energy shell $m$, thus manifestly solving the double counting problem of excitations responsible for the violation of the sum rules in all previous NRG spectral function approaches: The conventionally employed "patching" algorithms thus have become obsolete. Also, no even-odd oscillations can be observed. We believe that our novel approach provides the most accurate representation of local spectral functions for a given NRG input. It can be used in quantum impurity systems, at finite temperature as well as $T \rightarrow 0$. It is particular suitable for symmetry broken phases, for instance in an external magnetic field.

This significant improvement can have a major impact on the usage of the NRG as impurity solver for the dy- namical mean field theory (DMFT) $\frac{8.9}{2}$ In particular, for the description of symmetry broken phases such as the ferromagnetic Hubbard mode ${ }^{40}$ as well as orbital order in the two-band Hubbard model, the discrepancy between the local NRG order parameter and the one obtained from the spectral function often yields instabilities in iterating the DMFT equations. Moreover, very accurate spectral functions are needed for the application of the NRG to cluster DMFT approaches $\stackrel{10}{=}$ or periodic Anderson models which include crystal field effects 41 . Up to now such an accuracy could only be achieved with immense CPU time $\underline{\underline{42}}$

\section{Acknowledgments}

We acknowledge stimulating discussions with A. Weichselbaum which helped us clarifying that CFS can be applied to the evaluation of correlation functions rigorously such that inter-shell contributions are absent. After completion of this work we learned that Weichselbaum and von Delft independently had followed up on the same idea ${ }^{43}$ with a slightly different focus and a more generalized treatment of the density matrix. We further thank T. A. Costi for stimulating discussions, as well as A. Schiller for collaborations on related work.

This research was supported by the DFG through collaborative research grant SFB 602 (RP and TP) and project AN 275/5-1 (FBA). We acknowledge supercomputer support by the Gesellschaft für wissenschaftliche Datenverarbeitung Göttingen and the Norddeutsche Verbund für Hoch- und Höchstleistungsrechnen under project nip00015 (RP and TP), as well as the NIC, Forschungszentrum Jülich under project no. HHB000 (FBA).

\section{APPENDIX A: PROOF OF THE SPECTRAL SUM RULE}

By inserting the analytical expression (17) for the discrete spectral function into the integral (19)

$$
C=\oint \frac{d z}{2 \pi i} G_{A, B}(z)=\sum_{\alpha=i, i i, i i i} \oint \frac{d z}{2 \pi i} G_{A, B}^{\alpha}(z)
$$

yields the following three contributions

$$
\begin{aligned}
C= & \sum_{l, l^{\prime}} A_{l, l^{\prime}}(N) B_{l^{\prime}, l}(N)\left(e^{-\beta E_{l}^{N}}-s e^{-\beta E_{l^{\prime}}^{N}}\right) \\
& +\sum_{m=m_{\min }}^{N-1} \sum_{l} \sum_{k, k^{\prime}} B_{l, k^{\prime}}(m) \rho_{k^{\prime}, k}^{\mathrm{red}}(m) A_{k, l}(m) \\
& -s \sum_{m=m_{\text {min }}}^{N-1} \sum_{l} \sum_{k, k^{\prime}} A_{l, k^{\prime}}(m) \rho_{k^{\prime}, k}^{\mathrm{red}}(m) B_{k, l}(m) .
\end{aligned}
$$


By formally defining the "reduced density matrix" for the last Wilson shell as

$$
\rho_{k k^{\prime}}^{r e d}(N)=\delta_{k, k^{\prime}} \frac{e^{-\beta E_{k}^{N}}}{Z},
$$

the first term in A2 can be included into the second and third. Then the second reads

$$
\begin{aligned}
C_{2}= & \sum_{m=m_{m i n}}^{N} \sum_{l^{\prime}} \sum_{k, k^{\prime}} B_{l^{\prime}, k^{\prime}}(m) \rho_{k^{\prime}, k}^{\mathrm{red}}(m) A_{k, l^{\prime}}(m) \\
= & \sum_{m=m_{\text {min }}}^{N} \sum_{l^{\prime} e^{\prime \prime}} \sum_{k, e} \sum_{k^{\prime}, e^{\prime}}\left\langle l^{\prime}, e^{\prime \prime} ; m|B| k^{\prime}, e^{\prime} ; m\right\rangle \\
& \times\left\langle k^{\prime}, e^{\prime} ; m|\hat{\rho}| k, e ; m\right\rangle\left\langle k, e ; m|A| l^{\prime}, e^{\prime \prime} ; m\right\rangle
\end{aligned}
$$

In this step, we used the definition of the reduced density matrix and made use of the fact that the local operators $A$ and $B$ are independent of the environment variables $e, e^{\prime}, e^{\prime \prime}$. Due to the form of the density operator, $\left(1_{m}^{+}+\right.$ $\left.1_{m}^{-}\right) \rho\left(1_{m}^{+}+1_{m}^{-}\right)=1_{m}^{+} \rho 1_{m}^{+}$holds for $m<N$. For $N=m$, $1_{N}^{-}$spans the complete Fock space and the projection $1_{m}^{-} \rho 1_{m}^{-}$contains all contributions at $m=N$. Therefore, the contribution $C_{2}$ yields

$$
\begin{array}{r}
C_{2}=\sum_{m=m_{m i n}}^{N} \sum_{l^{\prime} e^{\prime \prime}}\left\langle l^{\prime}, e^{\prime \prime} ; m|B \rho A| l^{\prime}, e^{\prime \prime} ; m\right\rangle \\
=\operatorname{Tr}[\rho A B] .
\end{array}
$$

We can perform the same calculation for the third term in (A2) and the contribution stemming from the $(-s)$ part of the first term at $m=N$ to derive

$$
C_{3}=-s \operatorname{Tr}[\rho B A] .
$$

To this end, the total contribution is given by

$$
C=\operatorname{Tr}[\rho A B]-s \operatorname{Tr}[\rho B A]=\operatorname{Tr}\left[\rho[A, B]_{s}\right]
$$

We thus have proven that our Green's function fulfills the spectral sum rule exactly. Thus, any deviations for this sum rule in the broaden spectral function stems solemnly from the accuracy of the numerical $\omega$ integration.

\section{APPENDIX B: OCCUPATION SUM RULE}

We now want to discuss the accuracy of our approach for the generalized occupancy $n_{B, A}$ defined as

$$
n_{B, A}=\oint \frac{d z}{2 \pi i} f_{s}(z) G_{A, B}(z),
$$

where $f_{s}(z)=[\exp (\beta z)-s]^{-1}$. As always throughout the paper, this definition comprises bosonic and fermionic Green's functions. Specializing to the local spin-dependent fermionic spectral function, i.e. $A=f_{\sigma}$ and $B=f_{\sigma}^{\dagger}$, yields the occupational sum rule (20) as presented in section IID
Inserting the Green's function (17) into the expression (B1) and performing the contour integration yields the following three contributions:

$$
\begin{aligned}
n_{B, A}= & \sum_{l, l^{\prime}} \frac{e^{-\beta E_{l}}}{Z} B_{l, l^{\prime}}(N) A_{l^{\prime}, l}(N) \\
& +\sum_{m=m_{\min }}^{N-1} \sum_{l} \sum_{k, k^{\prime}} \frac{B_{l, k^{\prime}}(m) \rho_{k^{\prime}, k}^{\mathrm{red}}(m) A_{k, l}(m)}{e^{\beta\left(E_{l}^{m}-E_{k}^{m}\right)}-s} \\
& +\sum_{m=m_{\min }}^{N-1} \sum_{l} \sum_{k, k^{\prime}} \frac{A_{l, k^{\prime}}(m) \rho_{k^{\prime}, k}^{\mathrm{red}}(m) B_{k, l}(m)}{1-s e^{\beta\left(E_{k}^{m}-E_{l}^{m}\right)}} .
\end{aligned}
$$

Adding and subtracting

$$
\sum_{m=m_{m i n}}^{N-1} \sum_{l} \sum_{k, k^{\prime}} \frac{A_{l, k^{\prime}}(m) \rho_{k^{\prime}, k}^{\mathrm{red}}(m) B_{k, l}(m)}{e^{\beta\left(E_{l}^{m}-E_{k}^{m}\right)}-s}
$$

leads to

$$
\begin{aligned}
n_{B, A}= & \sum_{l, l^{\prime}} \frac{e^{-\beta E_{l}}}{Z} B_{l, l^{\prime}}(N) A_{l^{\prime}, l}(N) \\
& +\sum_{m=m_{m i n}}^{N-1} \sum_{l} \sum_{k, k^{\prime}} A_{l, k^{\prime}}(m) \rho_{k^{\prime}, k}^{\mathrm{red}}(m) B_{k, l}(m) \\
& +\delta n_{B, A} \\
= & \operatorname{Tr}[\rho B A]+\delta n_{B, A}
\end{aligned}
$$

using the same identities as in appendix A Finally, the error $\delta n_{B, A}$ of the occupation sum rule is given by

$$
\begin{aligned}
\delta n_{B, A}= & \sum_{m=m_{m i n}}^{N-1} \sum_{l, k, k^{\prime}} \rho_{k^{\prime}, k}^{\mathrm{red}}(m) \\
& \times \frac{B_{l, k^{\prime}}(m) A_{k, l}(m)-A_{l, k^{\prime}}(m) B_{k, l}(m)}{e^{\beta\left(E_{l}^{m}-E_{k}^{m}\right)}-s} .
\end{aligned}
$$

To estimate the size of this error, let us note that the excitation energies $E_{l}^{m}-E_{k}^{m}$ appearing in $\delta n_{B, A}$ are positive, because $l$ labels a discarded and $k$ a kept state. Excitations between two discarded states never contribute as well as excitations between to kept states. The smallest energy difference possible is given by the energy of the first discarded minus the energy of the last kept state. Even though this energy difference might be small, the smallness of the matrix element of the reduced density matrix suppresses this contribution. We are thus left with energy differences which are of the order $D_{m}$ for $m<N$. The density matrix $\hat{\rho}$, on the other hand, is restricted to the last iteration, i.e., to be consistent with the fundamental assumption of the NRG, $\exp \left[\beta\left(E_{l}^{m}-E_{k}^{m}\right)\right] \gg 1$ for $m<N$. Thus, the denominator in $\delta n_{B, A}$ becomes exponentially large and hence $\delta n_{B, A}$ exponentially suppressed with decreasing temperature. Note that this also means, that for $T \rightarrow 0$ the occupation sum rule will be obeyed exactly, too. For finite $T$ we must expect, and indeed find (see section IC), 
deviations from this sum rule with increasing temperature. Our numerical results show, however, that even for high temperatures these deviations stay below the per mille level.

\section{APPENDIX C: IMAGINARY TIME GREEN'S FUNCTIONS}

The textbook definition of the imaginary time Green's function is given by

$$
\begin{aligned}
G_{A, B}(\tau) & =-\operatorname{Tr}[\hat{\rho} T(A(\tau) B)] \\
& =\left\{\begin{array}{cc}
-\operatorname{Tr}\left[\hat{\rho} e^{\tau H} A e^{-\tau H} B\right] & ; \tau>0 \\
-s \operatorname{Tr}\left[\hat{\rho} B e^{\tau H} A e^{-\tau H}\right] & ; \tau<0
\end{array}\right.
\end{aligned}
$$

where again $s=1$ for Bosonic operators $A, B$ and $s=$ -1 for Fermionic operators. It is straight forward to show that the analytic continuation $G_{A, B}(z)$ of the exact Matsubara Green's function $G_{A, B}(i \omega)$

$$
G_{A, B}(i \omega)=\int_{0}^{\beta} d \tau e^{i \omega \tau} G_{A, B}(\tau),
$$

where $\omega=\pi(2 n+1) / \beta$ for Fermionic and $\omega=2 \pi n / \beta$ for Bosonic Green's functions, is identical to the Laplace transformed exact retarded Green's function (9) with $z=$ $i \omega$. We can perform the same steps for $G_{A, B}(\tau)$ as in section IIC In this case, however, the terms $-s[z+$ $\left.E_{l}^{m}-E_{k}^{m}\right]^{-1}$ in $G^{i i}(z)$ and $\left[z+E_{k}^{m}-E_{l}^{m}\right]^{-1}$ in $G^{i i i}(z)$ must be replaced by

$$
\frac{\exp \left(\beta\left(E_{k}^{m}-E_{l}^{m}\right)-s\right.}{i \omega+E_{l}^{m}-E_{k}^{m}}
$$

and

$$
\frac{1-s \exp \left[\beta\left(E_{k}^{m}-E_{l}^{m}\right)\right]}{i \omega+E_{k}^{m}-E_{l}^{m}} .
$$

At first sight, the two Green's functions are not equal in our approach. However, as discussed in appendix $B$, the Boltzmann factors $\exp \left[\beta\left(E_{k}^{m}-E_{l}^{m}\right)\right]$ can be neglected for $m<N$. This is justified by the NRG assumption that the density operator is well approximated by its contribution of the last Wilson shell, i.e. Eq. (7). Therefore, both Green's functions are identical within the NRG approximation.
1 A. C. Hewson, The Kondo Problem to Heavy Fermions (Cambridge Press, Cambridge UK, 1993).

2 M. A. Kastner, Rev. Mod. Phys. 64, 849 (1992).

3 D. Goldhaber-Gordon, H. Shtrikman, D. Mahalu, D. Abusch-Magder, U. Meirav, and M. Kastner, Nature 391, 156 (1998).

4 H. C. Manoharan, C. P. Lutz, and D. M. Eigler, Nature 403, 512 (2000).

5 O. Agam and A. Schiller, Phys. Rev. Lett. 86, 484 (2001).

${ }^{6}$ P. W. Anderson, Phys. Rev. 124, 41 (1961).

7 A. J. Leggett, S. Chakravarty, A. T. Dorsey, and M. P. A. Fisher, Rev. Mod. Phys. 59, 1 (1987).

8 T. Pruschke, M. Jarrell, and J. K. Freericks, Adv. in Phys. 42, 187 (1995).

9 A. Georges, G. Kotliar, W. Krauth, and M. J. Rozenberg, Rev. Mod. Phys. 68, 13 (1996), for a review on the DMFT.

10 T. Maier, M. Jarrell, T. Pruschke, and M. H. Hettler, Rev. Mod. Phys. 77, 1027 (2005).

11 K. G. Wilson, Rev. Mod. Phys. 47, 773 (1975).

12 H. R. Krishna-murthy, J. W. Wilkins, and K. G. Wilson, Phys. Rev. B 21, 1003 (1980).

13 H. R. Krishna-murthy, J. W. Wilkins, and K. G. Wilson, Phys. Rev. B 21, 1044 (1980).

14 D. L. Cox and A. Zawadowski, Advances in Physics 47, 599 (1998), for a review on the multi-channel models.

15 R. Bulla, T. A. Costi, and D. Vollhardt, Phys. Rev. B 64, 045103 (2001).

16 W. Hofstetter, Phys. Rev. Lett. 85, 1508 (2000).

17 T. Costi, A. C. Hewson, and V. Zlatic, J. Phys.: Condens. Matter 6, 2519 (1994).
18 R. Bulla, A. C. Hewson, and T. Pruschke, J. Phys.: Condens. Matter 10, 8365 (1998).

19 T. A. Costi, Phys. Rev. B 55, 3003 (1997).

${ }^{20}$ F. B. Anders and A. Schiller, Phys. Rev. Lett. 95, 196801 (2005).

21 F. B. Anders and A. Schiller (2006), cond-mat/0604517.

22 J. Kondo, Prog. Theor. Phys. 28, 864 (1962).

${ }^{23}$ F. B. Anders, Phys. Rev. B 71, 121101(R) (2005).

24 D. M. Cragg and P. Lloyd, J. Phys. C.: Solid State Phys. 12, L215 (1979).

25 H. B. Pang and D. L. Cox, Phys. Rev. Lett. 44, 9454 (1991).

26 B. A. Jones and C. M. Varma, Phys. Rev. Lett. 58, 843 (1987).

27 B. A. Jones, C. M. Varma, and J. W. Wilkins, Phys. Rev. Lett. 61, 125 (1988).

28 O. Sakai, Y. Shimizu, and T. Kasuya, Solid State Commun. 75, 81 (1990).

29 O. Sakai and Y. Shimizu, J. Phys. Soc. Jpn. 61, 2333 (1992).

30 O. Sakai and Y. Shimizu, J. Phys. Soc. Jpn 61, 2348 (1992)

31 K. Ingersent, B. A. Jones, and J. W. Wilkins, Phys. Rev. Lett. 69, 2594 (1992).

32 T. Pruschke, Physica B: Cond. Mat. 359-361, 633 (2005).

33 R. Bulla, N. Tong, and M. Vojta, Phys. Rev. Lett. 91, 170601 (2003).

34 R. Bulla, H.-J. Lee, N.-H. Tong, and M. Vojta, Phys. Rev. B 71, 045122 (2005).

35 M. T. Glossop and K. Ingersent, Phys. Rev. Lett. 95, 67202 
(2005).

36 R. P. Feynman, Statistical Mechanics, A Set of Lectures (Benjamin, Reading, MA, 1972).

37 S. White, Phys. Rev. Lett. 69, 2863 (1992).

38 O. Sakai, Y. Shimizu, and T. Kasuya, J. Phys. Soc. Japan 58, 3666 (1989).

39 F. B. Anders, N. Grewe, and A. Lorek, Z. Phys. B 54, 293 (1991).

40 R. Zitzler, T. Pruschke, and R. Bulla, Eur. Phys. J. B 27, 473 (2002).

41 F. B. Anders and T. Pruschke, Phys. Rev. Lett. 96, 086404 (2006).

42 T. Pruschke and R. Bulla, Eur. Phys. J. B 44, 217 (2005).

43 A. Weichselbaum and J. von Delft, cond-mat/0607497 (2006). We recommend this paper for a discussion of detailed balance and possible extensions of the NRG density operator as well as broading procedures.
${ }^{44}$ We use the standard notation, by which the $N$-site chain contains the impurity degrees of freedom, as well as the first $N+1$ Wilson shells (labelled by $n=0, \cdots, N$.)

${ }^{45}$ In contrast to the standard NRG notations we consider $\mathcal{H}_{N}$ as dimensionfull Hamiltonian in order to kept the notation as simple as possible.

46 Local operators we call operators which act only on chain links up to $m<m_{\min }$. See a detailed discussion in Refs. 2021.

47 We can either directly sum the weights of the discrete raw NRG spectrum or perform a numerical integration after having broadened it. The former usually yields an order of magnitude better accuracy than the latter.

48 This in fact holds for all spectra calculated, i.e. with or without magnetic field, at $T=0$ and finite temperature!

${ }^{49}$ For our calculations, we choose $\tilde{b}=0.8$ and $\alpha=2$. 